\title{
Atributos químicos de solos manejados por usina sucroalcooleira situada na Nova
}

\section{Alta Paulista}

Chemical attributes of soils managed by a sugar and alcohol plant located in Nova Alta Paulista

Atributos químicos del suelos gestionados por una planta de azúcar y alcohol ubicada en Nova Alta

Paulista

Recebido: 17/02/2021 | Revisado: 23/02/2021 | Aceito: 26/02/2021 | Publicado: 06/03/2021

\author{
Maurício Dominguez Nasser \\ ORCID: https://orcid.org/0000-0002-4239-5958 \\ Agência Paulista de Tecnologia dos Agronegócios, Brasil \\ E-mail: mauricio.nasser@sp.gov.br \\ Camila Paula Rossetto Pescatori Jacon \\ ORCID: https://orcid.org/0000-0001-9670-1087 \\ Faculdade de Ciências Agronômicas, Brasil \\ Universidade Estadual Paulista, Botucatu, Brasil \\ E-mail: capescatorij@gmail.com \\ Mateus Batista Tavares \\ ORCID: https://orcid.org/0000-0002-7204-6126 \\ Cooperativa Agrícola Mista de Adamantina, Brasil \\ E-mail: mateusb.admin@gmail.com \\ Carolina Bugalho Kohori \\ ORCID: https://orcid.org/0000-0003-2078-3893 \\ Coordenadoria de Desenvolvimento Rural Sustentável, Brasil \\ E-mail: carolina.kohori@sp.gov.br \\ João Paulo Silva Ferreira \\ ORCID: https://orcid.org/0000-0003-4667-5962 \\ Agronomia do Centro Universitário de Adamantina, Brasil \\ E-mail: fjoao.agronomia@gmail.com \\ Augusto Zonta \\ ORCID: https://orcid.org/0000-0002-1702-4602 \\ Agência Paulista de Tecnologia dos Agronegócios, Brasil \\ E-mail: azonta@protonmail.com
}

\begin{abstract}
Resumo
A cana-de-açúcar é considerada uma matriz energética renovável e limpa, o que tem levado à expansão das áreas de cultivo. A grande quantidade de fitomassa produzida pela cultura exige o fornecimento adequado de água e nutrientes, dessa forma, o diagnóstico e o manejo da fertilidade do solo é de fundamental importância. A análise química do solo é uma das principais ferramentas para isso, pois, permite a quantificação dos atributos químicos do solo que servem como base para a aplicação de fertilizantes, corretivos e condicionadores de solos, possibilitando produção sustentável e economicamente viável. O presente trabalho teve o objetivo de avaliar os atributos químicos de solos manejados por usina sucroalcooleira na Nova Alta Paulista. Em 1120 amostras de solo foram analisadas o pH, CTC, saturação por bases e os teores de matéria orgânica e macronutrientes e, em 280 amostras foi analisado também o teor de micronutrientes. Os resultados foram apresentados graficamente sendo a distribuição dos valores expressa em porcentagem. Também foi calculada a média e o valor de maior frequência, representado pela moda. Verificou-se que de modo geral os solos avaliados apresentam baixo teor de matéria orgânica. Os resultados indicam que os solos são corrigidos frequentemente com aplicação de calcário e sugerem um monitoramento constante dos teores de K, S, B e Zn para que esses nutrientes possam ser fornecidos de forma criteriosa possibilitando a manutenção ou aumento da fertilidade do solo e da produtividade da cana-de-açúcar.
\end{abstract}

Palavras-chave: Cana-de-açúcar; Saccharum officinarum L.; Nutrição de plantas; Correção do solo; Adubação.

\begin{abstract}
Sugarcane is considered a renewable and clean energy matrix, which has led to the expansion of cultivated areas. The large amount of phytomass produced by the crop requires an adequate supply of water and nutrients, so the diagnosis and management of soil fertility is of fundamental importance. The chemical analysis of the soil is one of the main tools for this, as it allows the quantification of the chemical attributes of the soil that serve as a basis for the application of fertilizers, soil amendments and conditioners, enabling sustainable and economically viable production. This study aimed to evaluate the chemical attributes of soils managed by a sugar and alcohol plant in Nova Alta Paulista. In 1120 soil samples the $\mathrm{pH}, \mathrm{CTC}$, base saturation and the contents of organic matter and macronutrients
\end{abstract}


were analyzed, and in 280 samples the micronutrient content was also analyzed. The results were presented graphically with the distribution of values expressed as a percentage. The average and the most frequent value, represented by fashion, were also calculated. It was found that in general the soils evaluated have low content of organic matter. The results indicate that the soils are frequently corrected with the application of lime and suggest a constant monitoring of the $\mathrm{K}, \mathrm{S}, \mathrm{B}$ and $\mathrm{Zn}$ contents so that these nutrients can be carefully provided, allowing the maintenance or increase of soil fertility and productivity of sugar cane.

Keywords: Sugar cane; Saccharum officinarum L.; Plant nutrition; Soil correction; Fertilization.

\section{Resumen}

La caña de azúcar es considerada una matriz energética renovable y limpia, lo que ha propiciado la expansión de áreas cultivadas. La gran cantidad de fitomasa que produce el cultivo requiere un adecuado aporte de agua y nutrientes, por lo que el diagnóstico y manejo de la fertilidad del suelo es de fundamental importancia. El análisis químico del suelo es una de las principales herramientas para ello, ya que permite la cuantificación de los atributos químicos del suelo que serem de base para la aplicación de fertilizantes, en miendas y acondicionadores del suelo, posibilitando una producción sostenible y económicamente viable. Este estudio tuvo como objetivo evaluar los atributos químicos de suelos manejados por una planta de azúcar y alcohol en Nova Alta Paulista. En 1120 muestras de suelo se analizó el pH, CTC, saturación de bases y el contenido de materia orgánica y macronutrientes, y en 280 muestras también se analizó el contenido de micronutrientes. Los resultados se presentaron gráficamente con la distribución de valores expresada como porcentaje. También se calcularon la media y el valor más frecuente, representado por la moda. Se encontró que en general los suelos evaluados bajo contenido de materia orgánica. Los resultados indican que los suelos se corrigen frecuentemente con la aplicación de cal y sugieren un monitoreo constante de los contenidos de K, $\mathrm{S}$, B y Zn para que estos nutrientes puedan ser aportados de manera cuidadosa permitiendo el mantenimiento o aumento de la fertilidad y productividad del suelo de caña de azúcar.Incluir o resumo em espanhol.

Palabras clave: Caña de azúcar; Saccharum officinarum L.; Nutrición vegetal; Corrección de suelos; Fertilización.

\section{Introdução}

A produção mundial de açúcar foi aproximadamente $180 \mathrm{mi}$ de toneladas com destaque para o Brasil, Índia, União Europeia, Tailândia, China e Estados Unidos (FAOSTAT, 2018). O Brasil que é considerado o maior produtor de açúcar originado da cana-de-açúcar (Saccharum officinarum L.) cultiva aproximadamente 10 milhões de hectares e produz por hectare em média 77 toneladas dessa gramínea (CONAB, 2020). Associado ao açúcar e visando a economia mundial de baixo carbono, o Brasil e suas usinas sucroalcooleiras apostam na cana-de-açúcar como matriz energética renovável e limpa. Essa demanda faz expandir a produção em todo país e aumenta a rentabilidade dessa cultura tropical (Linnenluecke et al., 2019; Rodrigues et al., 2020).

O agronegócio cana-de-açúcar também está instalado na Nova Alta Paulista. Essa região de clima tropical do oeste Paulista localizada no espigão divisor Peixe-Aguapeí teve seu solo explorado de forma significativa. A pecuária e agricultura como o cultivo do café, algodão e recentemente a cana-de-açúcar substituíram a vegetação de mata Atlântica Continental e o Cerrado (Boin, 2000).

O cultivo da cana-de-açúcar considerado exploração agropecuária cria mudanças nos ecossistemas naturais, e interfere na dinâmica existente entre solo, cobertura vegetal e presença de microorganismos, alterando processos de acúmulo e ciclagem de nutrientes, agregação do solo e decomposição gradativa da matéria orgânica, provocando queda na fertilidade do solo e aumento da compactação (Lourente et al., 2011; Freitas et al., 2017). A enorme quantia de fitomassa produzida pela planta exige água e nutrientes, que quando são fornecidos de forma adequada possibilitam altas produtividades (Lacerda et al., 2019).

Nesse contexto da nutrição de cana-de-açúcar em regiões tropicais, torna-se relevante adotar tecnologias viáveis para diagnosticar a fertilidade de solos, e uma das principais é a utilização da análise química. Através de amostras de solo retiradas de forma criteriosa e posteriormente analisadas em laboratórios é possível quantificar o teor de macro e micronutrientes disponíveis e outras características que servem de ferramenta para o engenheiro agrônomo da agroindústria canavieira tomar decisões quanto ao manejo de fertilizantes, corretivos e condicionadores de solos visando uma produção vegetal viável economicamente e com respeito ao ecossistema da região de cultivo. 
Diante do exposto, objetivou-se avaliar os atributos químicos de solos manejados por usina sucroalcooleira situada na Nova Alta Paulista.

\section{Metodologia}

Este estudo baseou-se em análises químicas de solo realizadas no ano de 2018 em diferentes áreas cultivadas com cana-de-açúcar pela Usina Branco Peres Açúcar, Álcool e Energia, situada no município de Adamantina-SP, na região da Nova Alta Paulista. O clima da região é classificado por Koppen como Cwa, mesotérmico úmido, com verões quentes e invernos secos. A média anual de precipitação é de $1300 \mathrm{~mm}$ e a temperatura média de $22^{\circ} \mathrm{C}$, sendo janeiro o mês com média mais quente $\left(26^{\circ} \mathrm{C}\right)$ e junho o mês com média mais fria, $17^{\circ} \mathrm{C}$ (Cavichioli et al., 2018).

As amostras de solo foram coletadas na profundidade de 0 a $25 \mathrm{~cm}$ e analisadas quimicamente no Laboratório da Cooperativa Agrícola Mista de Adamantina (CAMDA) no ano de 2018, seguindo a metodologia proposta por Raij et al. (2001). Em 1120 amostras foram realizadas análises de $\mathrm{pH}\left(\mathrm{CaCl}_{2}\right)$, matéria orgânica $\left(\mathrm{g} \mathrm{dm}^{-3}\right), \mathrm{P}$ resina $\left(\mathrm{mg} \mathrm{dm}{ }^{-3}\right), \mathrm{K}, \mathrm{Ca}, \mathrm{Mg}$, CTC $\left(\right.$ mmolc $\left.\mathrm{dm}^{-3}\right)$, saturação por bases (V\%) e S $\left(\mathrm{mg} \mathrm{dm}^{-3}\right)$ e, das 1120 amostras, em 280 foram analisados os micronutrientes: Fe, $\mathrm{Mn}, \mathrm{Zn}, \mathrm{Cu}$ e B $\left(\mathrm{mg} \mathrm{dm}^{-3}\right)$.

Os dados foram interpretados conforme as classes de teores proposta por Raij (1996), com exceção dos dados da capacidade de troca catiônica (CTC) e matéria orgânica (MO) que foram interpretados segundo Sobral et al. (2015).

Os resultados foram apresentados em gráficos sendo a distribuição dos valores de cada atributo do solo expressa em porcentagem. Também foi calculada a média e o valor de maior frequência, a moda. Foi utilizada a análise estatística descritiva, através do programa Microsoft Excel 2007 (Zorzetti et al., 2014; Pereira et al., 2018).

\section{Resultados e Discussão}

Na Figura 1 é possível observar que $21 \%$ dos solos avaliados o $\mathrm{pH}$ foi inferior a 5,0, indicando que estes solos apresentam potencial de resposta à calagem. No entanto, $49 \%$ apresentam $\mathrm{pH}$ dentro da faixa considerada adequada para maioria das culturas, entre 5,5 e 6,5, quando os nutrientes estão mais disponíveis para as plantas (Taiz \& Zeiger, 2013; Prezotti \& Guarçoni, 2013) e o valor da moda indica que há maior frequência de pH 5,7, indicando grande frequência de solos adequados ao cultivo dentro daqueles avaliados. Em levantamento da fertilidade de solo do estado de SP realizado pelo IPNI, a média de pH no município de Adamantina foi de 5,0, abaixo da observada neste estudo (Prochnow et al., 2018). Essa diferença pode ser devida ao maior número de amostras avaliado neste ensaio e ao manejo adequado de correção do solo adotado pela usina onde este estudo foi realizado. 
Figura 1 - Faixa de teores de $\mathrm{pH}\left(\mathrm{CaCl}_{2}\right)$, CTC $\left(\right.$ mmolc $\left.\mathrm{dm}^{-3}\right)$ e saturação por bases (V\%) de solos manejados por usina sucroalcooleira localizada em Adamantina-SP (1120 análises).

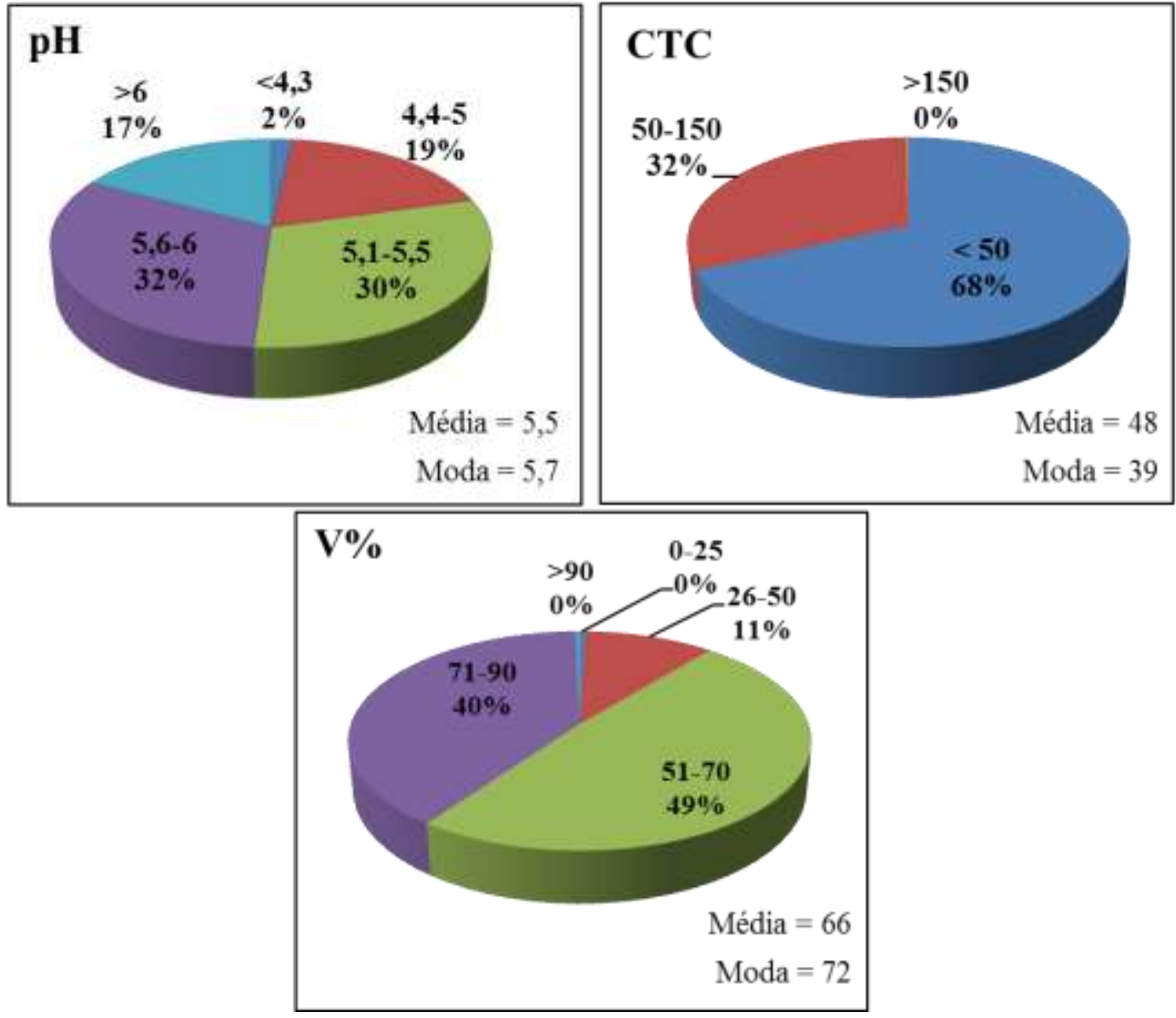

Fonte: Autores.

Quanto à CTC, 68\% dos solos avaliados apresentam valores abaixo de $50 \mathrm{mmolc} \mathrm{dm}^{-3}$, sendo $39 \mathrm{mmolc} \mathrm{dm}^{-3} \mathrm{o} \mathrm{valor}$ mais frequente nos solos avaliados, valor classificado como baixo por Sobral et al. (2015). A CTC de um solo representa a quantidade total de cátions retidos à sua superfície em condição permutável $\left(\mathrm{Ca}^{2+}+\mathrm{Mg}^{2+}+\mathrm{K}^{+}+\mathrm{H}^{+}+\mathrm{Al}^{3+}\right)$, segundo Ronquim (2020) e está diretamente relacionada à sua formação e ao seu teor de matéria orgânica. Os solos tropicais são normalmente formados por minerais como a caulinita que apresenta baixa CTC (Raij, 1991). Segundo Salomão (1994), as principais classes de solos encontradas no Oeste Paulista são o Latossolo Vermelho-Amarelo, Argissolo Vermelho-Amarelo e Argissolo Vermelho, que apresentam textura média ou média/arenosa, o que justifica os baixos valores de CTC observados. Além de a CTC representar a quantidade de cátions que um solo é capaz de reter, também indica a graduação da capacidade de liberar esses nutrientes retidos (Raij, 1991; Ronquim, 2020), portanto solos com baixa CTC conseguiriam manter a liberação de nutrientes por um período mais curto que os solos com CTC mais elevada. Como esses solos apresentam menor capacidade de retenção de água e nutrientes, torna-se importante realizar adubações e calagens de forma parcelada, evitando-se perdas por lixiviação, principalmente dos nutrientes mais móveis no solo como o nitrogênio e o potássio (Primavesi, 2006; Ronquim, 2020), garantindo melhor aproveitamento destes pelas plantas. Cabe ressaltar que a CTC é inalterável em curto prazo, porém a proporção dos cátions que a ocupam pode ser alterada (Raij, 2011). Se a maior parte da CTC de um solo está ocupada por cátions como $\mathrm{Ca}, \mathrm{Mg}$ e $\mathrm{K}$, pode-se dizer que esse solo está adequado para a nutrição das plantas. Mas, se a CTC está ocupada em grande parte por cátions potencialmente tóxicos como $\mathrm{H}^{+}$e $\mathrm{Al}^{3+}$, considera-se este um solo pobre (Ronquim, 2020).

A parte da CTC ocupada por $\mathrm{Ca}, \mathrm{Mg}$ e $\mathrm{K}$ é representada pela saturação por bases (V\%). Estudos mostram que os 
valores de V\% e o pH da camada arável de um solo estão relacionados positivamente (Raij, 1991), como observado nos solos avaliados neste estudo. Apenas 11\% apresentam saturação por bases considerada baixa (menor que 50\%) e há maior frequência de solos com saturação por bases de $72 \%$, considerado alto e adequado ao cultivo (Raij, 1996). Dessa forma, pode-se inferir que o manejo que vem sendo realizado pelos produtores da região, sob orientação de técnicos capacitados tem sido assertivo quanto à correção e manutenção dos valores de pH e saturação por bases dos solos.

Outro componente importante do solo é seu teor de matéria orgânica (Figura 2). O manejo preconizando seu aumento ao longo do tempo pode contribuir para a elevação da CTC e consequentemente da fertilidade do solo. Observa-se que nesta região $82 \%$ dos solos apresenta teor de MO inferior a $15 \%$, condizente com os tipos de solo e a textura arenosa, predominante na região (Raij et al., 1996; Sobral et al., 2015).

Observa-se também que o teor de $\mathrm{S}$ dos solos da região é baixo, $70 \%$ apresentam teor inferior a $4 \mathrm{mg} \mathrm{dm}^{-3}$. $\mathrm{O}$ enxofre é absorvido pelas plantas na forma de sulfato, forma que não é retida por muitos solos, sendo facilmente lixiviado e muitas vezes acumulado nas camadas mais profundas. Esse deslocamento do $\mathrm{S}$ em profundidade é acentuado pela elevação do $\mathrm{pH}$ do solo. O enxofre é encontrado no solo predominantemente na forma orgânica, portanto a capacidade do solo em suprir a demanda das plantas por esse nutriente está diretamente ligada ao seu teor de MO (Tiecher et al., 2012). Assim, os solos da região avaliada, arenosos, com baixo teor de MO também apresentam baixo teor de S. O manejo adequado da palhada da canade-açúcar pode contribuir para elevar o teor de $\mathrm{MO}$ dos solos e consequentemente a disponibilidade de nutrientes, a retenção de água e o desenvolvimento das plantas.

Dos solos analisados, $47 \%$ apresentaram teor médio de P, $20 \%$ teor alto ou muito alto e $33 \%$ teor baixo ou muito baixo. Segundo Malavolta (2006), o fósforo é o nutriente que mais limita a produção vegetal no Brasil, sendo um grande desafio a garantia de elevação da disponibilidade desse elemento na solução do solo. Comparado a outros macronutrientes, o fósforo é requerido em menor quantidade pela planta, os solos brasileiros são conhecidos pela carência em fósforo, devido principalmente ao material de origem e a forte interação deste elemento com o solo (Raij, 1991). 
Figura 2. Faixa de teores de matéria orgânica $(\mathrm{MO})\left(\mathrm{g} \mathrm{dm}^{-3}\right)$, enxofre $(\mathrm{S})$ e fósforo $(\mathrm{P})\left(\mathrm{mg} \mathrm{dm}^{-3}\right)$ em solos manejados por usina sucroalcooleira localizada em Adamantina-SP (1120 análises).
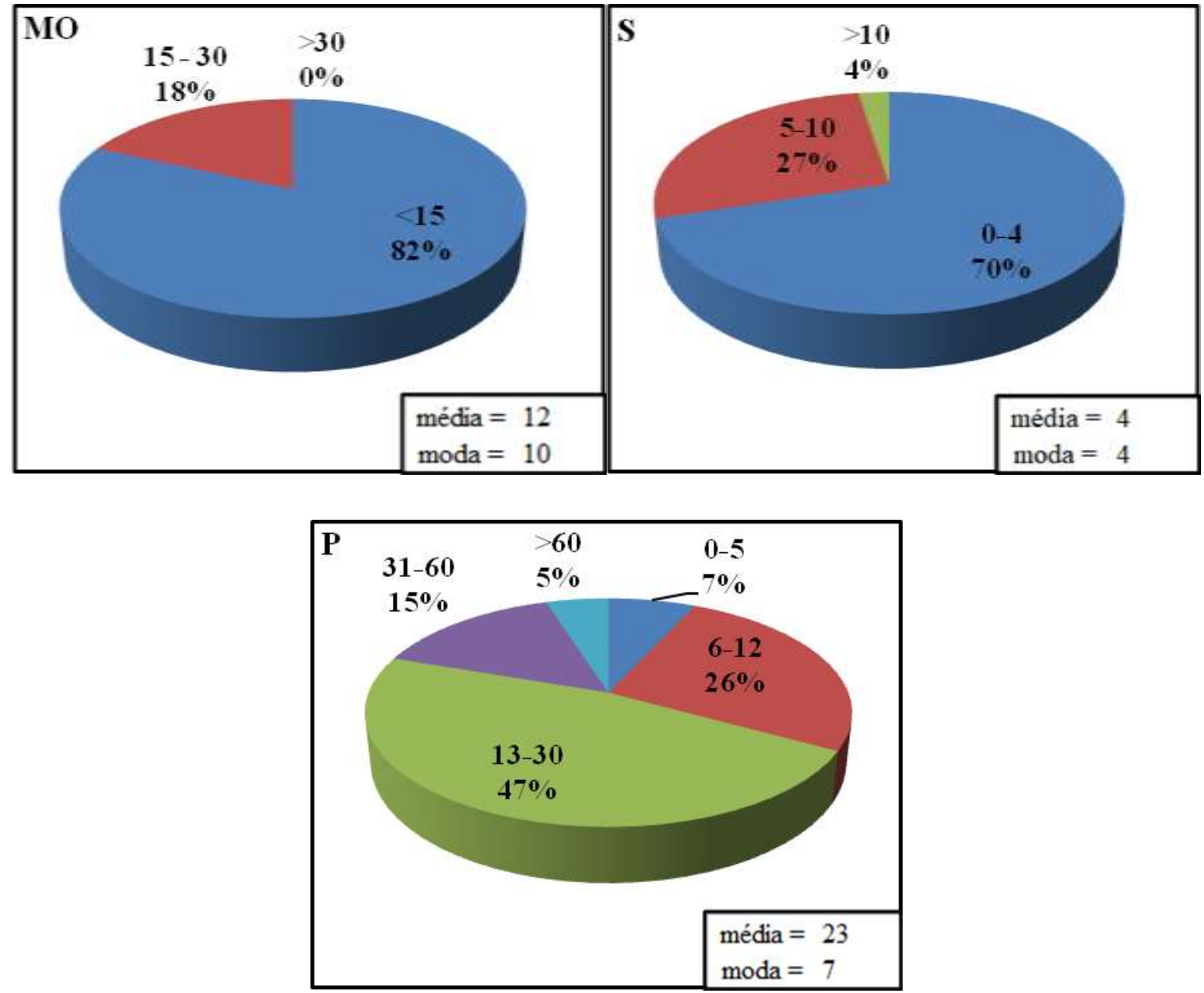

Fonte: Autores.

Na Figura 3 são apresentadas as porcentagens de teores de $\mathrm{K}$, Ca e Mg nos solos avaliados. Para o K, 48\%

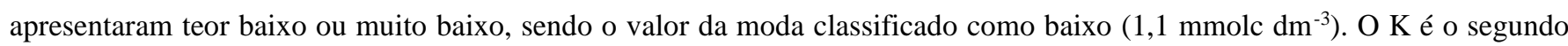
nutriente mineral requerido em maior quantidade pelas espécies vegetais e desempenha papel importante no crescimento da cana-de-açúcar, na produtividade de colmos e na produtividade de açúcar, especialmente em solos com baixos teores do nutriente (Otto et al., 2010). No entanto, pode ser intensamente lixiviado no perfil do solo, dependendo da quantidade de chuva, da dose de nutriente e da textura do solo, entre outros fatores (Rosolem et al., 2006). No setor sucroalcooleiro é prática comum a aplicação do K ser realizada de uma única vez, na ocasião do plantio (Lana et al., 2004) o que favorece a lixiviação e pode causar a salinização da região que recebe o fertilizante, podendo causar toxidez às raízes das plantas (Otto et al., 2010). Recomenda-se a aplicação de K parceladamente, conforme as plantas se desenvolvem, visando reduzir perdas no sistema soloplanta e aumentar a eficiência de utilização desse nutriente. Entretanto, no manejo da aplicação desse nutriente deve ser considerado o tipo de solo da área, pois alguns fatores podem interferir na relação solo-planta-nutriente, alterando a resposta da cana-de-açúcar à fertilização potássica. Em solos com maior CTC, o K em solução pode ser adsorvido às cargas negativas do solo e tornar-se menos disponível às plantas em relação a solos com baixa CTC. Além disso, problemas com lixiviação de K são mais intensos em solos arenosos, com baixa CTC. Lana et al. (2004) e Orlando Filho et al. (1993), observaram maior probabilidade de resposta ao parcelamento do $\mathrm{K}$ nos solos de textura médio-arenosa enquanto Casagrande et al. (1983) relataram que solos de textura mais argilosa e com maior CTC o parcelamento pode até mesmo limitar a produtividade da 
cana-de-açúcar devido ao elevado poder-tampão do solo em K. Esses resultados dão suporte à recomendação de parcelamento da adubação potássica para cana-de-açúcar cultivada em solos de textura arenosa no Estado de São Paulo, conforme Spironello et al. (1997).

Observa-se teor de Ca considerado alto em $98 \%$ dos solos avaliados e apenas $12 \%$ destes apresentaram baixo teor de Mg. Os teores de Ca e Mg observados podem ser decorrentes do manejo adequado da calagem e consequente fornecimento de cálcio e magnésio, já que a maior parte dos solos também apresentou valores de V\% de médio a alto. Além disso, se

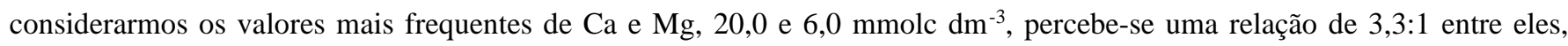
valor semelhante à concentração desses nutrientes no calcário dolomítico, comumente utilizado.

Figura 3. Faixa de teores de potássio, cálcio e magnésio (mmolc $\mathrm{dm}^{-3}$ ) em análises de solos manejados por usina sucroalcooleira localizada em Adamantina-SP (1120 análises).
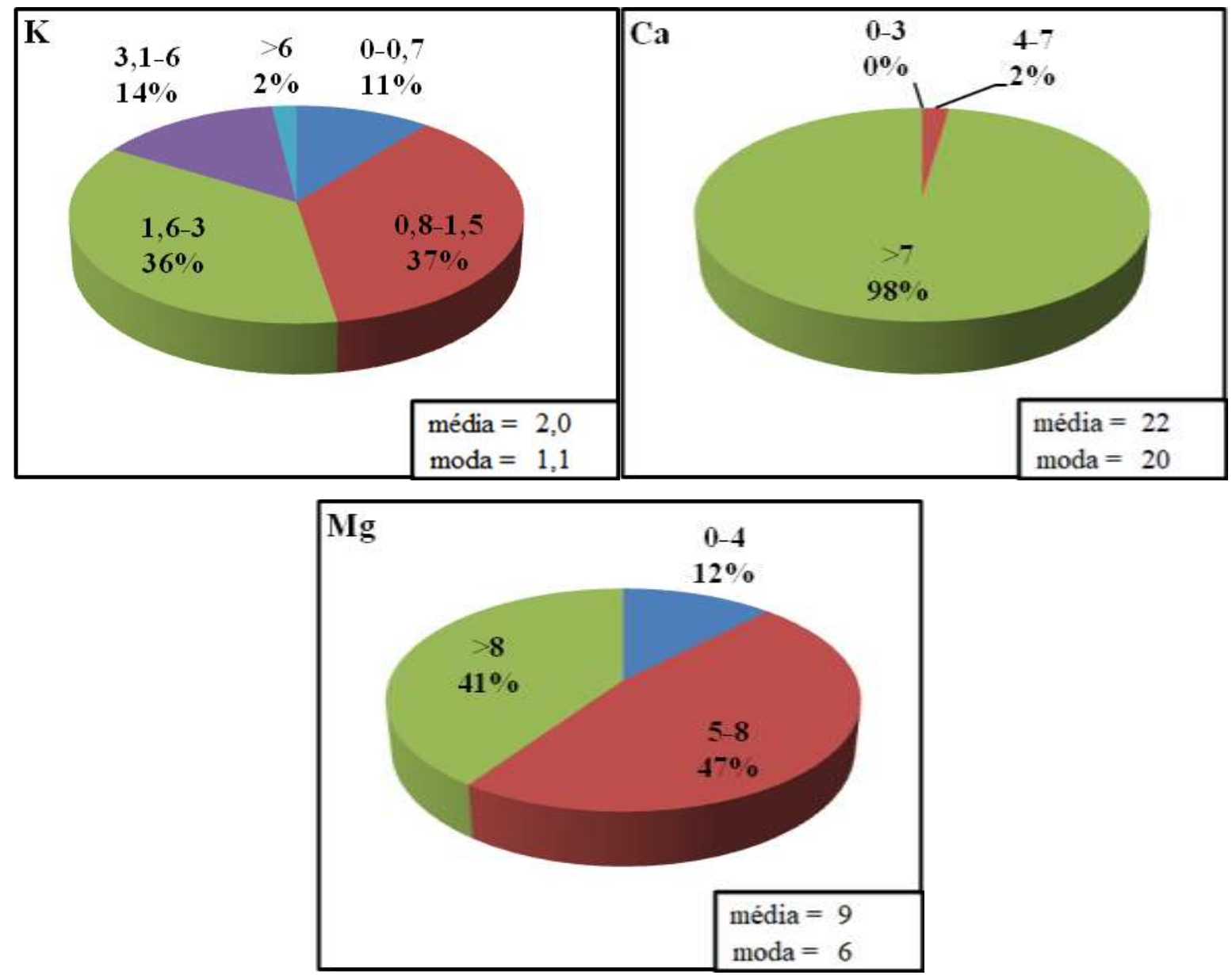

Fonte: Autores.

Nas Figuras 4 e 5 são apresentados os teores de micronutrientes nos solos avaliados. Apenas $5 \%$ dos solos apresentaram teores de $\mathrm{Zn}$ disponível maiores que $1,2 \mathrm{mg} \mathrm{dm}^{-3}$, classificado como alto e $70 \%$ dos solos apresentou teor classificado como baixo, assim como o valor mais frequentemente observado, $0,2 \mathrm{mg} \mathrm{dm}^{-3}$. Com relação ao boro, $90 \%$ dos solos avaliados estavam com teores baixos, menores que $0,2 \mathrm{mg} \mathrm{dm}^{-3}$, concordando com Rosolem e Bíscaro (2007) que afirmaram que solos de textura arenosa, pobres em matéria orgânica, tendem a apresentar baixa disponibilidade de B.

Os teores de Fe são classificados como altos em $88 \%$ dos solos, assim como o valor mais frequentemente observado nas análises realizadas, $25 \mathrm{mg} \mathrm{dm}^{-3}$. Para Mn, apenas $23 \%$ dos solos apresentaram teores classificados como médios por Raij et 
al. (1996), não tendo sido observados solos com teores baixos. Dos solos avaliados, 69\% apresentaram teores de cobre classificados como médios e apenas $2 \%$ apresentaram teores baixos.

Para o cultivo de cana-de-açúcar é recomendado por Raij et al. (1996), a aplicação de Zn quando os teores forem menores que $0,5 \mathrm{mg} \mathrm{dm}^{-3}$ e Cu quando os teores forem menores que $0,2 \mathrm{mg} \mathrm{dm}^{-3}$, não havendo recomendação para a aplicação dos demais micronutrientes. No entanto observa-se que mesmo com a recomendação de aplicação, o Zn encontra-se com teores baixos na maioria dos solos e o $\mathrm{Cu}$ com teores médios.

Sabe-se que os micronutrientes têm sua disponibilidade reduzida com a elevação do pH do solo e que altas doses de fertilizantes fosfatados pode reduzir a solubilidade do Zn (Raij, 1991). Segundo Demattê (2005) a aplicação de micronutrientes na cultura da cana é uma prática pouco utilizada, no entanto, deve-se considerar a recomendação de aplicação. Adorna et al. (2013) concluíram que a aplicação de Zn e B propiciaram aumento na produtividade de colmos e açúcar em solo arenoso e com baixo teor do nutriente. Outros estudos associando o B ao uso de maturadores químicos indicaram estreita correlação entre o fornecimento do nutriente na pré-colheita da cana-de-açúcar e o aumento do teor de sacarose das plantas (Leite, 2010; Siqueira, 2014). Considerando a deficiência de Zn e B na maior parte dos solos do presente trabalho, a aplicação de fertilizantes contendo esses elementos como os termofosfatos associado com micronutrientes poderia ser uma alternativa.

Figura 4. Faixa de teores de zinco e boro $\left(\mathrm{mg} \mathrm{dm}^{-3}\right)$ em solos manejados por usina sucroalcooleira localizada em AdamantinaSP.

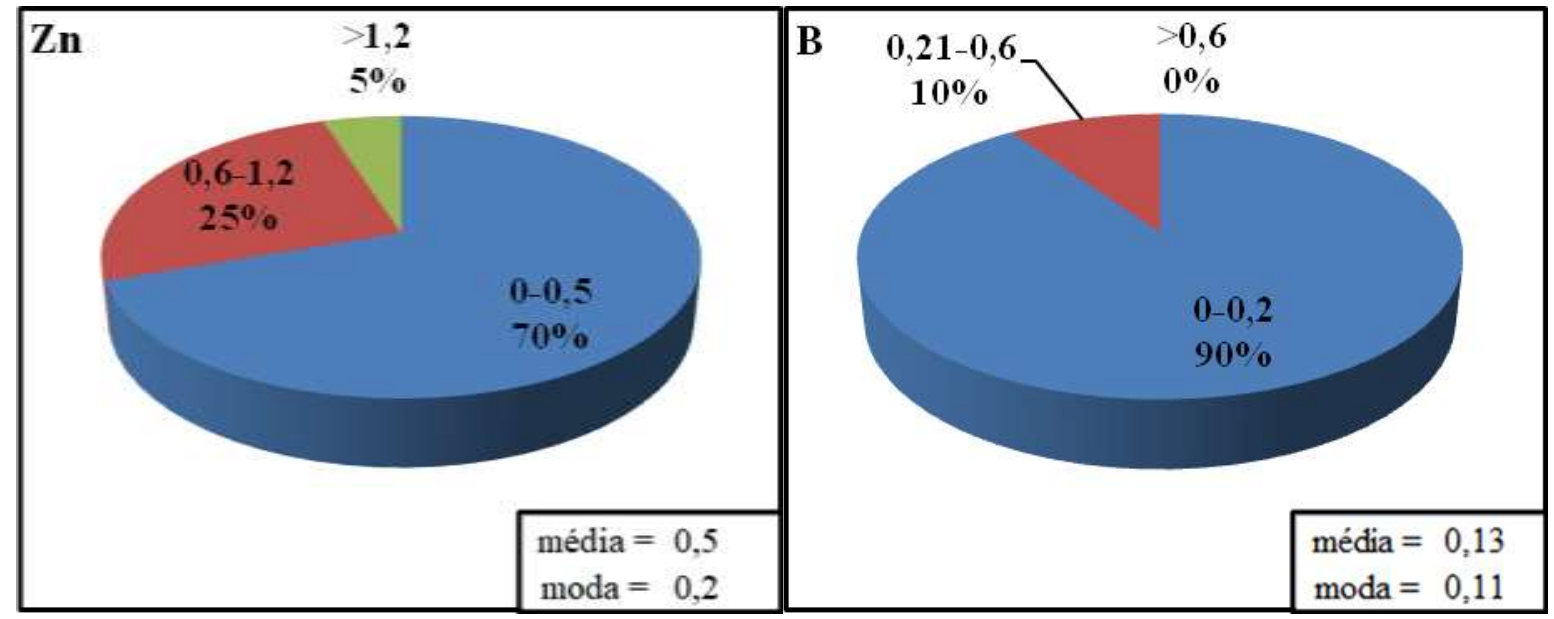

Fonte: Autores. 
Figura 5. Faixa de teores e respectiva porcentagem de ferro, manganês e cobre $\left(\mathrm{mg} \mathrm{dm}^{-3}\right)$ em solos manejados por usina sucroalcooleira localizada em Adamantina-SP.

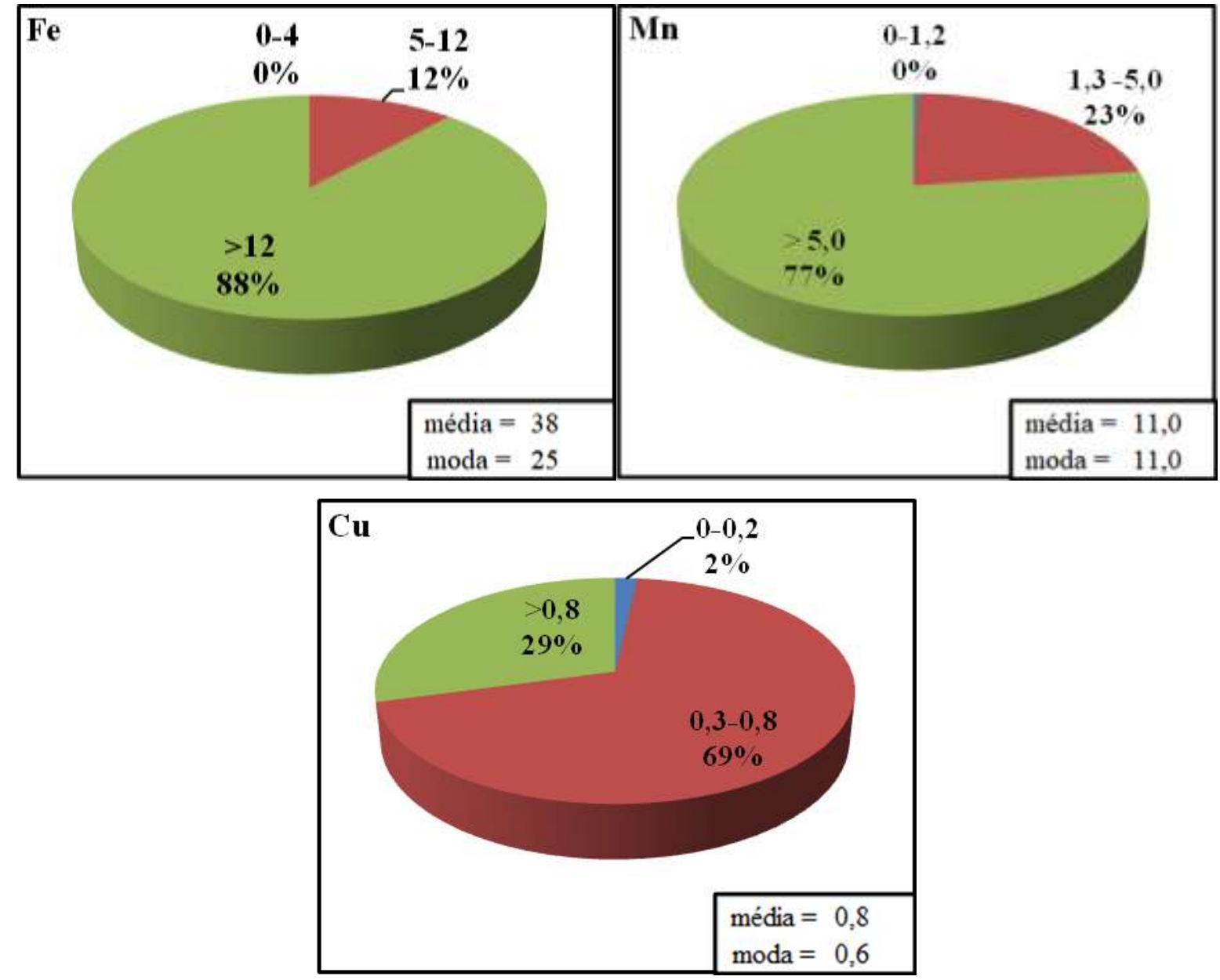

Fonte: Autores.

\section{Conclusão}

Os resultados obtidos demonstram que os solos manejados por usina sucroalcooleira situada na Nova Alta Paulista apresentam, de modo geral, baixa presença de matéria orgânica e indicam uso frequente de calagem. Além disso, sugerem o monitoramento constante dos teores de potássio, enxofre, boro e zinco, permitindo que sejam aplicados de forma criteriosa para a manutenção ou aumento da fertilidade do solo e da produtividade da cana-de-açúcar.

\section{Referências}

Adorna, J. C., Crusciol, C. A. C., \& Rossato, O. B. (2013). Fertilization with filter cake and micronutrients in plant cane. Revista Brasileira de Ciência do Solo, 37(3), 649-657.

Boin, M. N. (2000). Chuvas e erosões no oeste paulista: uma análise climatológica aplicada (Tese de Doutorado em Geociências e Meio Ambiente). - Instituto de Geociências e Ciências Exatas, Universidade Estadual Paulista, Rio Claro, SP, Brasil.

Casagrande, J. C., Zambello Junior, E., \& Orlando Filho, J. (1983). Fracionamento da adubação nitrogenada e potássica em cana-planta no Estado de São Paulo. Saccharum, 28, 43-48.

Cavichioli, J. C., Nasser, M. D., \& Vitorino, R. A. (2018). Sobrevivência e desempenho produtivo de maracujazeiro amarelo enxertado por encostia com raiz dupla. Revista de Ciências Agroambientais, 16(1), 25-30.

Conab - Companhia Nacional de Abastecimento. (2020). Acompanhamento da safra brasileira: cana-de-açúcar. 7 - SAFRA 2020/21 N. 3 - Terceiro Levantamento. Brasília. p. 1-62. https:/www.conab.gov.br/component/k2/item/download/34870_e1c52a336b53ca05c29824831da3c9e9

Demattê, J. L. I. (2005). Cultura da cana-de-açúcar - Recuperação e manutenção da fertilidade dos solos. Informaç̃os Agronômicas, 111, 1-24. 
FAOSTAT. Food and Agriculture Organization of the United Nations - Statistics Division. (2018). Crops processed. https:// http://www.fao.org/faostat/en/\#data/QD

Freitas, L., Oliveira, I. A., Silva, L. S., Frare, J. C. V., Filla, V. A., \& Gomes, R. P. (2017). Indicadores da qualidade química e física do solo sob diferentes sistemas de manejo. Revista Unimar Ciências, 26(1-2), 8-25.

Lacerda, A. R. S., Souza, A. R., Santos, T. M., Clemente, J. M., Duarte, A. R., \& Machado, M. G. (2019). Produtividade da cana-de-açúcar em resposta a adubação NPK em diferentes épocas. Humanidades \& Tecnologia em Revista (FINOM), 18(1), 1-7.

Lana, R. M. Q., Zanão Júnior, L. A., Korndorfer, G. H., \& Maciel Júnior, V. A. (2004). Parcelamento da adubação potássica na cana-planta. STAB - Açúcar, Álcool e Subprodutos, 23, 28-31.

Leite, G. H. P. (2010). Maturadores associados à boro e silício aplicados via foliar em cana-de-açúcar (Saccharum spp.) (Tese de Doutorado). Faculdade de Ciências Agronômicas, Universidade Estadual Paulista, Botucatu, SP, Brasil.

Linnenluecke, M. K., Han, J., Zheyao, P., \& Smith, T. (2019). How markets will drive the transition to a low carbon economy. Economic Modelling, 77(1), $42-54$.

Lourente, E. R. P., Mercante, F. M., Alovisi, A. M. T., Gomes, C. F., Gasparini, A. S., \& Nunes, C. M. (2011). Atributos microbiológicos, químicos e físicos de solo sob diferentes sistemas de manejo e condições de cerrado. Pesquisa Agropecuária Tropical, 41(1), 20-28.

Malavolta, E. (2006). Manual de nutrição mineral de plantas: Ceres.

Orlando Filho, J., Rossetto, R., \& Geraldi, R. N. (1993). Adubação potássica em cana-de-açúcar: II - Análise química do solo e diagnose foliar. Anais do Congresso Nacional da Stab - Sociedade dos Técnicos Açucareiros e Alcooleiros do Brasil, Águas de São Pedro, SP, Brasil, 5.

Otto, R., Vitti, G. C., \& Luz, P. H. de C. (2010). Manejo da adubação potássica na cultura da cana-de-açúcar. Revista Brasileira de Ciência do Solo, 34(4), 1137-1145. http://www.scielo.br/scielo.php?script =sci_arttext\&pid=S0100-06832010000400013\&lng=en\&nrm=iso.

Pereira, A. S., Shitsuka, D. M., Parreira, F. J., \& Shitsuka, R. (2018). Metodologia da pesquisa científica. UFSM. https://repositorio.ufsm.br/bitstream/handle/1/15824/Lic_Computacao_Metodologia-Pesquisa-Cientifica.pdf?sequence=1.

Prezotti, L. C., \& Guarçoni, A. (2013). Guia de interpretação de análise de solo e foliar: Incaper.

Primavesi, A. (2006). Manejo ecológico do solo: a agricultura em regiões tropicais. (18a ed.): Nobel.

Prochnow, L. I., da Cunha, J. F., Jorgetto, L. O., Cantarella, H., Lavorenti, E. L., \& Fernandes, D. M. (2018). Increasing soil fertility in the state of São Paulo. Informações Agronômicas, 161, 1-8. https://www.npct.com.br/npctweb/ npct.nsf/article/lfs-sp.

Raij, B van. (2011). Fertilidade do solo e manejo de nutrientes: International Plant Nutrition Institute.

Raij, B. van. (1991). Fertilidade do solo e adubação. Piracicaba, CERES, Potafos.

Raij, B. V., Andrade, J. C., Cantarella, H., \& Quaggio, J. A. (Eds.). (2001). Análise química para avaliação da fertilidade de solos tropicais: Instituto Agronômico.

Raij, B. V., Cantarella, H., Quaggio, J. A., \& Furlani, A. M. C. (1996). Recomendações de adubação e calagem para o Estado de São Paulo (2ª ed.): Instituto Agronômico de Campinas. (Boletim Técnico IAC, 100).

Rodrigues, J. dos S., Silva, P. C., Costa, A. R. da, Gomes, L. F., Silva, F. de F., Soares, J. A. B., \& Ferreira, J. B. G. (2020). Sugarcane productivity with waste water application from the sucroenergetic industry. Research, Society and Development, 9(5), e162953167. https://doi.org/10.33448/rsd-v9i5.3167

Ronquim, C. C. (2020). Conceitos de fertilidade do solo e manejo adequado para as regiões tropicais. (2 $2^{\mathrm{a}}$.ed.): Embrapa Territorial. https://www.infoteca.cnptia.embrapa.br/infoteca/bitstream/doc/1128267/1/5840.pdf

Rosolem, C. A., \& Bíscaro, T. (2007). Adsorção e lixiviação de boro em Latossolo Vermelho-Amarelo. Pesquisa Agropecuária Brasileira, 42(10), 1473-1478.

Rosolem, C. A., Santos, F. P., Foloni, J. S. S., \& Calonego, J. C. (2006). Potássio no solo em consequência da adubação sobre a palha de milheto e chuva simulada. Pesquisa Agropecuária Brasileira, 41(6), 1033-1040.

Salomão, F. X. T. (1994). 'Solos do Arenito Bauru'. In V. P. Pereira, M. E. Cruz, \& M. C. P. Cruz (Orgs.), Solos altamente suscetíveis à erosão (pp. 51-68): Sociedade Brasileira de Ciência do Solo.

Siqueira, G. F. (2014). Aplicação de boro e maturadores na pré-colheita da cana-de-açúcar em início e final de safra (Tese Doutorado). Faculdade de Ciências Agronômicas, Universidade Estadual Paulista, Botucatu, SP, Brasil.

Sobral, L. F., Barreto, M. C. V., Silva, A. J., \& Anjos, J. L. (2015). Guia prático para interpretação de resultados de análise de solo. Embrapa Tabuleiros Costeiros: Aracaju, 13 p. (Documentos, 206).

Spironello, A., Raij, B. van, Penatti, C. P., Cantarella, H., Morelli, J. L., Orlando Filho, J., Landell, M. G. A., \& Rosseto, R. (1997). Outras culturas industriais. In: Raij, B. Van., Raij, H., Cantarella, J. A. Quaggio, \& A. M. C. Furlani (Coords.), Recomendações de adubação e calagem para o Estado de São Paulo (Boletim Técnico, 100) (pp. 233-239): Instituto Agronômico.

Taiz, L., \& Zeiger, E. (2013). Fisiologia vegetal. (5a ed.): Artmed. 
Research, Society and Development, v. 10, n. 3, e6510313053, 2021

(CC BY 4.0) | ISSN 2525-3409 | DOI: http://dx.doi.org/10.33448/rsd-v10i3.13053

Tiecher, T., Santos, D. R., Rasche, J. W. A., Brunetto, G., Mallmann, F. J. K., \& Piccin, R. (2012). Resposta de culturas e disponibilidade de enxofre em solos com diferentes teores de argila e matéria orgânica submetidos à adubação sulfatada. Bragantia, 71(4), 518-527. https://www.scielo.br/scielo.php?pid=S0006$87052012000400010 \&$ script=sci_abstract\&tlng=pt.

Zorzetti, J., Neves, P. M. O. J., Santoro, P. H., \& Constanski, K. C. (2014). Conhecimento sobre a utilização segura de agrotóxicos por agricultores da mesorregião do Norte Central do Paraná. Semina: Ciências Agrárias, 35(4), 2415-2427. 